\title{
WEIGHTED YOUNG-TYPE INEQUALITIES ON LOCALLY COMPACT GROUPS
}

\author{
Frédéric Morneau-Guérin ${ }^{2}$
}

\begin{abstract}
We obtain an extension of Young's convolution inequality in weighted Lebesgue spaces of measurable functions defined on locally compact groups. Our result provides a unified treatment of a theorem of Klein and Russo extending the classical Young's inequality to locally compact groups, and a theorem of Biswas and Swanson generalizing Young's inequality to weighted Lebesgue spaces on locally compact abelian groups.
\end{abstract}

AMS Mathematics Subject Classification (2010): 43A15; 43A20; 22D15

Key words and phrases: Convolution operator; Young's inequality for convolution; Weighted spaces; Locally compact groups

\section{Introduction}

Given a locally compact (Hausdorff) group $G$ with a left Haar measure $\lambda$ (i.e. the unique - up to a positive multiplicative constant - left-invariant Radon measure on $G$ ) the space $L_{p}(G)$ with $1 \leq p \leq \infty$ is the usual Lebesgue space of all (equivalence classes) of complex valued $\lambda$-measurable functions $f$ on $G$ verifying

$$
(1 \leq p<\infty) \quad\|f\|_{p}:=\left(\int_{G}|f(x)|^{p} d_{L} \lambda(x)\right)^{1 / p}<\infty,
$$

or

$$
\|f\|_{\infty}:=\inf \{M \geq 0:|f(x)| \leq M \lambda \text {-almost everywhere }\}<\infty .
$$

Let $f, g: G \rightarrow \mathbb{C}$ be two $\lambda$-measurable functions. Their convolution $f * g:$ $G \rightarrow \mathbb{C}$ is the function defined by the formula

$$
(f * g)(x)=\int_{G} f(y) g\left(y^{-1} x\right) d_{L} \lambda(y) .
$$

However for $f * g$ to be well-defined, we need to impose conditions on $f$ and $g$ to ensure that (1.1) makes sense for almost all $x \in G$. A common choice is to require that $f, g \in L_{1}(G)$. It is easy to check that under this hypothesis

\footnotetext{
${ }^{1}$ This research was carried out at Département de mathématiques et de statistique, Université Laval. It was supported by a scholarship granted by Fonds de recherche Nature et technologies.

${ }^{2}$ Département Éducation, Université TÉLUQ, e-mail: frederic.morneau-guerin@teluq.ca
} 
$f * g$ is well-defined, that it belongs to $L_{1}(G)$ and that it verifies the following inequality :

$$
\|f * g\|_{1} \leq\|f\|_{1}\|g\|_{1} .
$$

This inequality turns out to be a particular case of the following classical result.

Theorem 1.1. (YOUnG'S INEQUALITY FOR CONVOLUTION) For all locally unimodular group $G$, if $p, q, r \in[1, \infty]$ are such that $\frac{1}{p}+\frac{1}{q}=\frac{1}{r}+1$, then for all $f \in L_{p}(G)$ and $g \in L_{q}(G)$ the convolution $f * g$ exists and belongs to $L_{r}(G)$. Moreover

$$
\|f * g\|_{r} \leq\|f\|_{p}\|g\|_{q} .
$$

Due to the pivotal role played by Theorem 1.1 in the study of the convolution operator, considerable attention was devoted to investigating its various improvements and generalizations. The program encompassed efforts aimed at strengthening equation 1.2 to a sharp form, i.e.

$$
\|f * g\|_{r} \leq c_{p, q}\|f\|_{p}\|g\|_{q},
$$

with $c_{p, q}<1$ (see for instance [2, 6, 11]), as well as investigations directed at obtaining various other Young-type inequalities, i.e. inequalities of the form

$$
\|f * g\|_{Z} \leq\|f\|_{X}\|g\|_{Y}
$$

where $X, Y$ and $Z$ are function spaces, $f \in X$, and $g \in Y$.

\section{Weighted $L_{p}$-spaces}

Given a locally compact group $G$, a weight function on $G$ is a strictly positive $\lambda$-measurable function defined on $G$. Given $1 \leq p \leq \infty$, the weighted $L_{p}$-space with weight $w$ is defined as follows :

$$
L_{p}(G, w):=\left\{f: G \rightarrow \mathbb{C} \mid\|f\|_{p, w}<\infty\right\},
$$

where $\|f\|_{p, w}:=\|w f\|_{p}$. Hence $L_{p}(G, w)$ is a Banach space with the norm $\|f\|_{p, w}$.

Weights and weighted function spaces play a distinguished role in numerical mathematics and have several concrete applications in computer science, engineering and statistics (time-frequency analysis, Gabor frames, wavelet frames, sampling theory, etc.); see for instance [10, 15, 16, 14, 12. Additionally, weighted function spaces appear naturally in functional analysis and operator theory. Such spaces have proved instrumental in questions of factorization as well as in the interpolation theory; see [5, 8.

Following [4], for $p, q, r \in[1, \infty]$, we define $\mathcal{Y}_{G}(p, q, r)$ as the set of all triplets of weight functions $\left(w_{1}, w_{2}, w_{3}\right)$ for which a Young-type inequality holds, i.e. there exists a positive constant $C=C\left(p, q, r, w_{1}, w_{2}, w_{3}\right)$ with the property that

$$
\|f * g\|_{r, w_{3}} \leq C\|f\|_{p, w_{1}}\|g\|_{q, w_{2}}
$$


for all $f \in L_{p}\left(G, w_{1}\right)$ and $g \in L_{q}\left(G, w_{2}\right)$.

The pursuit of criterions for membership in the class $\mathcal{Y}_{G}(p, q, r)$ can be traced at least as far back as the late 1950's; see for instance [7. The list of authors who sought necessary and/or sufficient conditions for inequalities analogous to 1.3 to hold true is too long to be mentioned exhaustively here. But let us mention the work of Wermer [29, Nikol'skii [27], Kerlin \& Lambert [19], Feichtinger 9], Grabiner [13. Kerman \& Sawyer 20], Abtahi, Nasr-Isfahani \& Rejali [1, Kuznetsova [23, 24, 25, 26, 22, Biswas \& Swanson [4, Toft, Johansson, Pilipović \& Teofanov [28, and Guo, Chen, Fan \& Zhao [17.

The main purpose of this note is to present an extension to any locally compact group of a theorem of Biswas \& Swanson identifying sufficient conditions ensuring that the triplet $\left(w_{1}, w_{2}, w_{3}\right)$ of weight functions on a locally compact abelian group $G$ belongs to $\mathcal{Y}_{G}(p, q, r)$.

\section{Interpolation theorem}

In this section we state, for reference, the version of the Riesz-Thorin interpolation theorem that will be used later in our proofs.

\section{Theorem 3.1. (RIESZ-THORIN INTERPOLATION THEOREM)}

Let $1 \leq p_{0}, p_{1}, q_{0}, q_{1} \leq \infty$ with $p_{0} \neq p_{1}$ and $q_{0} \neq q_{1}$. If $T$ is a linear operator boundedly mapping $L_{p_{0}}(U, d \mu)$ and $L_{p_{1}}(U, d \mu)$ into $L_{q_{0}}(V, d v)$ and $L_{q_{1}}(V, d v)$ respectively, then $T: L_{p_{\theta}}(U, d \mu) \rightarrow L_{q_{\theta}}(V, d v)$ with norm estimate

$$
\|T\|_{L_{p_{\theta}} \rightarrow L_{q_{\theta}}} \leq\|T\|_{L_{p_{0}} \rightarrow L_{q_{0}}}^{1-\theta}\|T\|_{L_{p_{1}} \rightarrow L_{q_{1}}}^{\theta}
$$

for each $\theta \in[0,1]$, provided that

$$
\frac{1}{p_{\theta}}=\frac{1-\theta}{p_{0}}+\frac{\theta}{p_{1}}, \quad \frac{1}{q_{\theta}}=\frac{1-\theta}{q_{0}}+\frac{\theta}{q_{1}} .
$$

Proof. See [3, Theorem 1.1.1.]

\section{Main results}

Before initiating the presentation of the main results, we shall briefly recall some important properties of the modular function of a locally compact group.

Given $\mu$ a left Haar measure on some locally compact group $G$, then for every $x \in G$, the measure $\mu_{x}$ defined by $\mu_{x}(E)=\mu(E x)$, for every Borel set $E$, is also a left Haar measure. Hence, by uniqueness, there must exist a positive number $\Delta(x)$ such that $\mu_{x}=\Delta(x) \mu$. The map $\Delta: G \rightarrow(0, \infty)$ thus defined, called the modular function on $G$, is continuous, positive throughout $G$, independent of the choice of $\mu$, and it satisfies the following multiplicative identity

$$
\Delta(x y)=\Delta(x) \Delta(y)
$$

for all $x, y \in G$. 
An important feature of the modular function is that it determines when the Haar measure is both left and right translation invariant. Indeed the left Haar measure $\mu$ on $G$ is also right translation invariant if and only if $\Delta \equiv 1$, in which case $G$ is said to be unimodular.

For a more thorough and comprehensive presentation of the properties of the modular function, one can consult [18].

Our first result deals with those triplets of indices satisfying the same relation as in the classical Young's inequality for convolution. We proceed along the lines set out by Klein \& Russo in the unweighted case; see 21.

Theorem 4.1. Let $G$ be a locally compact group with a left Haar measure $\lambda$ and of modular function $\Delta$. Assume that $p, q, r \in[1, \infty]$ satisfy the following relation

$$
\frac{1}{p}+\frac{1}{q}=\frac{1}{r}+1 .
$$

Let $w_{1}, w_{2}, w_{3}$ be weight functions on $G$ satisfying, for some positive constant $C$, the following inequality

$$
w_{3}(x) \leq C w_{1}(y) w_{2}\left(y^{-1} x\right),
$$

for $\lambda$-almost every $x, y \in G$. Then, for all $f \in L_{p}\left(G, w_{1}\right)$ and $g \in L_{q}\left(G, w_{2}\right)$, the convolution $f * g \Delta^{\frac{1}{q}-\frac{1}{r}}$ exists and belongs to $L_{r}\left(G, w_{3}\right)$. Moreover,

$$
\left\|f * g \Delta^{\frac{1}{q}-\frac{1}{r}}\right\|_{r, w_{3}} \leq C\|f\|_{p, w_{1}}\|g\|_{q, w_{2}} .
$$

It is worth noting that under our assumptions on $p, q, r$ we have

$$
\frac{1}{q}-\frac{1}{r}=1-\frac{1}{p}=\frac{1}{p^{\prime}}
$$

where $p^{\prime}$ denotes the Hölder conjugate of $p$. We chose to hold on to the notation $\frac{1}{q}-\frac{1}{r}$ in the statement of Theorem 4.1 for the sake of consistency with the notation of Theorem 4.1. However, in order to make the notation less cluttered in the proof of Theorem 4.4. we will write $\frac{1}{p^{\prime}}$ rather than $\frac{1}{q}-\frac{1}{r}$.

It is helpful first to establish the following two results.

Lemma 4.2. Under the assumption of Theorem 4.1 we have, for all $p \in[1, \infty]$,

$$
\left\|f * g \Delta^{\frac{1}{p^{\prime}}}\right\|_{p, w_{3}} \leq C\|f\|_{p, w_{1}}\|g\|_{1, w_{2}}
$$

Proof. If $p=\infty$, it follows from th.2 that for $\lambda$-almost every $x, y \in G$ we have

$$
\begin{aligned}
|f * g(x) \Delta| & \leq \frac{C}{w_{3}(x)} \int_{G} w_{1}(y)|f(y)| w_{2}\left(y^{-1} x\right)\left|g\left(y^{-1} x\right)\right| \Delta\left(y^{-1} x\right) d_{L} \lambda(y) \\
& \leq \frac{C\|f\|_{\infty, w_{1}}}{w_{3}(x)} \int_{G} w_{2}\left(y^{-1} x\right)\left|g\left(y^{-1} x\right)\right| \Delta\left(y^{-1} x\right) d_{L} \lambda(y)
\end{aligned}
$$


Hence

$$
\|f * g \Delta\|_{\infty, w_{3}} \leq C\|f\|_{\infty, w_{1}}\|g\|_{1, w_{2}}
$$

If $p=1$ we have $p^{\prime}=\infty$ and it follows that $\Delta^{\frac{1}{p^{\prime}}} \equiv 1$. Then by 4.2 we have for $\lambda$-almost every $x, y \in G$

$$
|f * g(x)| \leq \frac{C}{w_{3}(x)} \int_{G} w_{1}(y)|f(y)| w_{2}\left(y^{-1} x\right)\left|g\left(y^{-1} x\right)\right| d_{L} \lambda(y)
$$

So, by Fubini's theorem, we have

$$
\begin{aligned}
\|f * g\|_{1, w_{3}} & =\int_{G} w_{3}(x)|f * g(x)| d_{L} \lambda(x) \\
& \leq C \int_{G} \int_{G} w_{1}(y)|f(y)| w_{2}\left(y^{-1} x\right)\left|g\left(y^{-1} x\right)\right| d_{L} \lambda(y) d_{L} \lambda(x) \\
& =C \int_{G} w_{1}(y)|f(y)| \int_{G} w_{2}\left(y^{-1} x\right)\left|g\left(y^{-1} x\right)\right| d_{L} \lambda(x) d_{L} \lambda(y) d_{L} \\
& =C \int_{G} w_{1}(y)|f(y)| \int_{G} w_{2}(x)|g(x)| d_{L} \lambda(x) d_{L} \lambda(y)
\end{aligned}
$$

Hence

$$
\|f * g\|_{1, w_{3}} \leq C\|f\|_{1, w_{1}}\|g\|_{1, w_{2}} .
$$

If $p \in(1, \infty)$, then by 4.2 and Hölder's inequality (see [18, Theorem 12.4]) we have for $\lambda$-almost every $x, y \in G$

$$
\begin{aligned}
& \left|f * g \Delta^{\frac{1}{p^{\prime}}}(x)\right| \\
\leq \quad & \frac{C}{w_{3}(x)} \int_{G} w_{1}(y)|f(y)| w_{2}\left(y^{-1} x\right)\left|g\left(y^{-1} x\right)\right| \Delta\left(y^{-1} x\right)^{\frac{1}{p^{\prime}}} d_{L} \lambda(y) \\
\leq \quad & \frac{C}{w_{3}(x)} \int_{G} w_{1}(y)|f(y)| w_{2}\left(y^{-1} x\right)^{\frac{1}{p}+\frac{1}{p^{\prime}}}\left|g\left(y^{-1} x\right)\right|^{\frac{1}{p}+\frac{1}{p^{\prime}}} \Delta\left(y^{-1} x\right)^{\frac{1}{p^{\prime}}} d_{L} \lambda(y) \\
\leq \quad & \frac{C}{w_{3}(x)}\left(\int_{G} w_{1}(y)^{p}|f(y)|^{p} w_{2}\left(y^{-1} x\right) \mid g\left(y^{-1} x\right) d_{L} \lambda(y)\right)^{1 / p} \\
& \cdot\left(\int_{G} w_{2}\left(y^{-1} x\right)\left|g\left(y^{-1} x\right)\right| \Delta\left(y^{-1} x\right) d_{L} \lambda(y)\right)^{1 / p^{\prime}} \\
= & \frac{C}{w_{3}(x)}\left(\int_{G} w_{1}(y)^{p}|f(y)|^{p} w_{2}\left(y^{-1} x\right)\left|g\left(y^{-1} x\right)\right| d_{L} \lambda(y)\right)^{1 / p}\|g\|_{1, w_{2}}^{1 / p^{\prime}}
\end{aligned}
$$


Therefore, by Fubini's theorem, we have

$$
\begin{aligned}
& \left\|f * g \Delta^{\frac{1}{p^{\prime}}}\right\|_{p, w_{3}}^{p} \\
= & \int_{G} w_{3}(x)^{p}\left|f * g \Delta^{\frac{1}{p^{\prime}}}(x)\right|^{p} d_{L} \lambda(x) \\
\leq \quad & C^{p}\|g\|_{1, w_{2}}^{p / p^{\prime}} \int_{G} \int_{G} w_{1}(y)^{p}|f(y)|^{p} w_{2}\left(y^{-1} x\right)\left|g\left(y^{-1} x\right)\right| d_{L} \lambda(y) d_{L} \lambda(x) \\
\leq \quad & C^{p}\|g\|_{1, w_{2}}^{p / p^{\prime}} \int_{G} w_{1}(y)^{p}|f(y)|^{p} \int_{G} w_{2}\left(y^{-1} x\right)\left|g\left(y^{-1} x\right)\right| d_{L} \lambda(x) d_{L} \lambda(y) \\
= & C^{p}\|g\|_{1, w_{2}}^{p / p^{\prime}} \int_{G} w_{1}(y)^{p}|f(y)|^{p} \int_{G} w_{2}(x)|g(x)| d_{L} \lambda(x) d_{L} \lambda(y) \\
= & C^{p}\|g\|_{1, w_{2}}^{p / p^{\prime}}\|g\|_{1, w_{2}}\|f\|_{p, w_{1}}^{p} \cdot
\end{aligned}
$$

But $\frac{p}{p^{\prime}}+1=p$. So, taking the $p$-th root, we get

$$
\left\|f * g \Delta^{\frac{1}{p^{\prime}}}\right\|_{p, w_{3}} \leq C\|f\|_{p, w_{1}}\|g\|_{1, w_{2}}
$$

Lemma 4.3. Under the assumption of Theorem 4.1 we have, for all $p \in[1, \infty]$,

$$
\left\|f * g \Delta^{\frac{1}{p^{\prime}}}\right\|_{\infty, w_{3}} \leq C\|f\|_{p, w_{1}}\|g\|_{p^{\prime}, w_{2}}
$$

Proof. If $p=1$ we have $p^{\prime}=\infty$ and it follows that $\Delta^{\frac{1}{p^{\prime}}} \equiv 1$. Therefore 4.2 implies that for $\lambda$-almost every $x, y \in G$ we have

$$
\begin{aligned}
|f * g(x)| & \leq \frac{C}{w_{3}(x)} \int_{G} w_{1}(y)|f(y)| w_{2}\left(y^{-1} x\right)\left|g\left(y^{-1} x\right)\right| d_{L} \lambda(y) \\
& =\frac{C\|f\|_{1, w_{1}}\|g\|_{\infty, w_{2}}}{w_{3}(x)}
\end{aligned}
$$

Hence

$$
\|f * g\|_{\infty, w_{3}} \leq C\|f\|_{1, w_{1}}\|g\|_{\infty, w_{2}}
$$

If $p=\infty$, then we can apply Lemma 4.2 and the result follows.

If $p \in(1, \infty)$, then by 4.2 and Hölder's inequality we have for $\lambda$-almost every $x, y \in G$

$$
\begin{aligned}
& \left|f * g \Delta^{\frac{1}{p^{\prime}}}(x)\right| \\
\leq & \frac{C}{w_{3}(x)} \int_{G} w_{1}(y)|f(y)| w_{2}\left(y^{-1} x\right)\left|g\left(y^{-1} x\right)\right| \Delta\left(y^{-1} x\right)^{\frac{1}{p^{\prime}}} d_{L} \lambda(y) \\
\leq \quad & \frac{C}{w_{3}(x)}\left(\int_{G} w_{1}(y)^{p}|f(y)|^{p} d_{L} \lambda(y)\right)^{1 / p} \\
& \cdot\left(\int_{G} w_{2}\left(y^{-1} x\right)^{p^{\prime}}\left|g\left(y^{-1} x\right)\right|^{p^{\prime}} \Delta\left(y^{-1} x\right) d_{L} \lambda(y)\right)^{1 / p^{\prime}} \\
= & \frac{C}{w_{3}(x)}\|f\|_{p, w_{1}}\|g\|_{p^{\prime}, w_{2} .}
\end{aligned}
$$


Hence

$$
\left\|f * g \Delta^{\frac{1}{p^{\prime}}}\right\|_{\infty, w_{3}} \leq C\|f\|_{p, w_{1}}\|g\|_{p^{\prime}, w_{2}}
$$

Proof. (of Theorem 4.1) For fixed $p \in[1, \infty]$ and $f \in L_{p}(G)$, it follows from Lemmas 4.2 and 4.3 that the application $T$ defined on simple functions by $g \mapsto f * g \Delta^{\frac{1}{p^{\prime}}}$ maps boundedly $L_{1}\left(G, w_{2}\right)$ and $L_{p^{\prime}}\left(G, w_{2}\right)$ respectively into $L_{p}\left(G, w_{3}\right)$ and $L_{\infty}\left(G, w_{3}\right)$ with norm less than or equal to $C\|f\|_{p, w_{1}}$ in both cases. Then, Theorem 3.1 implies that $T$ maps $L_{p_{\theta}}\left(G, w_{2}\right)$ boundedly into $L_{q_{\theta}}\left(G, w_{3}\right)$ for $\theta \in[0,1]$, where $p_{\theta}$ and $q_{\theta}$ are defined as

$$
\frac{1}{p_{\theta}}=\frac{1-\theta}{1}+\frac{\theta}{p^{\prime}} \quad \text { and } \quad \frac{1}{q_{\theta}}=\frac{1-\theta}{p}+\frac{\theta}{\infty} .
$$

Moreover, the following operator norm estimate holds :

$$
\begin{aligned}
\|T\|_{L_{p_{\theta}}\left(G, w_{2}\right) \rightarrow L_{q_{\theta}}\left(G, w_{3}\right)} & \leq\|T\|_{L_{1}\left(G, w_{2}\right) \rightarrow L_{p}\left(G, w_{3}\right)}^{1-\theta}\|T\|_{L_{p^{\prime}}\left(G, w_{2}\right) \rightarrow L_{\infty}\left(G, w_{3}\right)}^{\theta} \\
& \leq C\|f\|_{p, w_{1}}
\end{aligned}
$$

Hence, if we set $q:=p_{\theta}$ and $r:=q_{\theta}$, we get

$$
\left\|f * g \Delta^{\frac{1}{p^{\prime}}}\right\|_{r, w_{3}} \leq C\|f\|_{p, w_{1}}\|g\|_{q, w_{2}} .
$$

Our second result deals with those triplets of indices satisfying a relation slightly different than that of the classical Young's inequality. The proof is adapted from that of a theorem of Biswas \& Swanson; see [4].

Theorem 4.4. Let $G$ be a locally compact group with a left Haar measure $\lambda$ and of modular function $\Delta$. Assume that $p, q, r, t \in[1, \infty]$ satisfy

$$
1<t \leq \min \{p, q, r\} \leq \infty \quad \text { and } \quad \frac{1}{p}+\frac{1}{q}=\frac{1}{r}+\frac{1}{t},
$$

and that $t^{\prime}$ denotes the Hölder conjugate of $t$. Let $w_{1}, w_{2}, w_{3}$ be weight functions on $G$ satisfying, for some positive constant $C$, the following inequality

$$
\left(w_{1}^{-t^{\prime}} * w_{2}^{-t^{\prime}}\right)(x) \leq C^{t^{\prime}} w_{3}^{-t^{\prime}}(x) .
$$

for $\lambda$-almost every $x, y \in G$. Then for all $f \in L_{p}\left(G, w_{1}\right)$ and $g \in L_{q}\left(G, w_{2}\right)$ the convolution $f * g$ exists and belongs to $L_{r}\left(G, w_{3}\right)$. Moreover

$$
\left\|f * g \Delta^{\frac{1}{q}-\frac{1}{r}}\right\|_{r, w_{3}} \leq\|f\|_{p, w_{1}}\|g\|_{q, w_{2}} .
$$


Proof. We shall prove the theorem using division into cases. Throughout the proof, assume that $f \in L_{p}\left(G, w_{1}\right)$ and $g \in L_{q}\left(G, w_{2}\right)$.

CASE I. Suppose that $p=q=r=\infty$, wherefrom $t=\infty$ as well. We have $t^{\prime}=1$ and $\frac{1}{q}-\frac{1}{r}=0$ so $\Delta^{1 / q-1 / r} \equiv 1$. Thus, for $\lambda$-almost every $x, y \in G$

$$
\begin{aligned}
|f * g(x)| & \leq \int_{G} \frac{w_{1}(y)|f(y)| w_{2}\left(y^{-1} x\right)\left|g\left(y^{-1} x\right)\right|}{w_{1}(y) w_{2}\left(y^{-1} x\right)} d_{L} \lambda(y) \\
& \leq\|f\|_{\infty, w_{1}}\|g\|_{\infty, w_{2}}\left(w_{1}^{-1} * w_{2}^{-1}\right)(x) \\
& \leq\|f\|_{\infty, w_{1}}\|g\|_{\infty, w_{2}} C w_{3}^{-1}(x) .
\end{aligned}
$$

Therefore

$$
\|f * g\|_{\infty, w_{3}} \leq C\|f\|_{\infty, w_{1}}\|g\|_{\infty, w_{2}}
$$

CASE II. Suppose that $p=t<\infty$ and $q=r=\infty$. So once more we have $\frac{1}{q}-\frac{1}{r}=0$ and $\Delta^{1 / q-1 / r} \equiv 1$. By Hölder's inequality, we have for $\lambda$-almost every $x, y \in G$

$$
\begin{aligned}
& |f * g(x)| \\
\leq & \int_{G} \frac{w_{1}(y)|f(y)| w_{2}\left(y^{-1} x\right)\left|g\left(y^{-1} x\right)\right|}{w_{1}(y) w_{2}\left(y^{-1} x\right)} d_{L} \lambda(y) \\
\leq & \|g\|_{\infty, w_{2}} \int_{G} \frac{w_{1}(y)|f(y)|}{w_{1}(y) w_{2}\left(y^{-1} x\right)} d_{L} \lambda(y) \\
\leq & \|g\|_{\infty, w_{2}}\left(\int_{G} w_{1}(y)^{t}|f(y)|^{t} d_{L} \lambda(y)\right)^{1 / t} \\
& \cdot\left(\int_{G} \frac{1}{w_{1}(y)^{t^{\prime}} w_{2}\left(y^{-1} x\right)^{t^{\prime}}} d_{L} \lambda(y)\right)^{1 / t^{\prime}} \\
\leq & \|g\|_{\infty, w_{2}}\|f\|_{t, w_{1}}\left(w_{1}^{-t^{\prime}} * w_{2}^{-t^{\prime}}(x)\right)^{1 / t^{\prime}} \\
\leq & \|g\|_{\infty, w_{2}}\|f\|_{t, w_{1}}\left(C^{t^{\prime}} w_{3}^{-t^{\prime}}(x)\right)^{1 / t^{\prime}}
\end{aligned}
$$

Hence

$$
\|f * g\|_{\infty, w_{3}} \leq C\|f\|_{p, w_{1}}\|g\|_{\infty, w_{2}} .
$$

CAse III. Suppose that $q=t<\infty$ and $p=r=\infty$. By Hölder's inequality 
and (4.4) we have for $\lambda$-almost every $x \in G$

$$
\begin{aligned}
& \left|f * g \Delta^{\frac{1}{q}}(x)\right| \\
\leq & \int_{G} \frac{w_{1}(y)|f(y)| w_{2}\left(y^{-1} x\right)\left|g\left(y^{-1} x\right)\right| \Delta^{\frac{1}{q}}\left(y^{-1} x\right)}{w_{1}(y) w_{2}\left(y^{-1} x\right)} d_{L} \lambda(y) \\
\leq & \|f\|_{\infty, w_{1}} \int_{G} \frac{w_{2}\left(y^{-1} x\right)\left|g\left(y^{-1} x\right)\right| \Delta^{\frac{1}{q}}\left(y^{-1} x\right)}{w_{1}(y) w_{2}\left(y^{-1} x\right)} d_{L} \lambda(y) \\
\leq & \|f\|_{\infty, w_{1}}\left(\int_{G} w_{2}\left(y^{-1} x\right)^{t}\left|g\left(y^{-1} x\right)\right|^{t} \Delta^{\frac{t}{q}}\left(y^{-1} x\right) d_{L} \lambda(y)\right)^{1 / t} \\
& \cdot\left(\int_{G} \frac{1}{w_{1}(y)^{t^{\prime}} w_{2}\left(y^{-1} x\right)^{t^{\prime}}} d_{L} \lambda(y)\right)^{1 / t^{\prime}} \\
= & \|f\|_{\infty, w_{1}}\left(\int_{G} w_{2}\left(y^{-1} x\right)^{q}\left|g\left(y^{-1} x\right)\right|^{q} \Delta\left(y^{-1} x\right) d_{L} \lambda(y)\right)^{1 / q} \\
\leq & \quad \|\left(w_{1}^{-t^{\prime}} *\left\|_{\infty, w_{1}}\right\| g \|_{q, w_{2}}\left(C^{\prime}(x)\right)^{1 / t^{\prime}} w_{3}^{-t^{\prime}}(x)\right)^{1 / t^{\prime}}
\end{aligned}
$$

Therefore,

$$
\|f * g\|_{\infty, w_{3}} \leq C\|f\|_{\infty, w_{1}}\|g\|_{q, w_{2}} .
$$

CASE IV. Suppose that $p, q, t<\infty$ and $r=\infty$. By Hölder's inequality and 4.4 we have

$$
\begin{aligned}
& \left|f * g \Delta^{\frac{1}{q}}(x)\right| \\
\leq & \int_{G} \frac{w_{1}(y)|f(y)| w_{2}\left(y^{-1} x\right)\left|g\left(y^{-1} x\right)\right| \Delta^{\frac{1}{q}}\left(y^{-1} x\right)}{w_{1}(y) w_{2}\left(y^{-1} x\right)} d_{L} \lambda(y) \\
\leq & \left(\int_{G} w_{1}(y)^{t}|f(y)|^{t} w_{2}\left(y^{-1} x\right)^{t}\left|g\left(y^{-1} x\right)\right|^{t} \Delta^{\frac{t}{q}}\left(y^{-1} x\right) d_{L} \lambda(y)\right)^{1 / t} \\
& \cdot\left(\int_{G} \frac{1}{w_{1}(y)^{t^{\prime}} w_{2}\left(y^{-1} x\right)^{t^{\prime}}} d_{L} \lambda(y)\right)^{1 / t^{\prime}} \\
= & \left(\int_{G} w_{1}(y)^{t}|f(y)|^{t} w_{2}\left(y^{-1} x\right)^{t}\left|g\left(y^{-1} x\right)\right|^{t} \Delta^{\frac{t}{q}}\left(y^{-1} x\right) d_{L} \lambda(y)\right)^{1 / t} \\
& \cdot\left(w_{1}^{-t^{\prime}} * w_{2}^{-t^{\prime}}(x)\right)^{1 / t^{\prime}} \\
\leq & \left(\int_{G} w_{1}(y)^{t}|f(y)|^{t} w_{2}\left(y^{-1} x\right)^{t}\left|g\left(y^{-1} x\right)\right|^{t} \Delta^{\frac{t}{q}}\left(y^{-1} x\right) d_{L} \lambda(y)\right)^{1 / t} \\
& \cdot\left(C^{t^{\prime}} w_{3}^{-t^{\prime}}(x)\right)^{1 / t^{\prime}}
\end{aligned}
$$


Therefore

$$
\begin{aligned}
& w_{3}(x)\left|f * g \Delta^{\frac{t}{q}}(x)\right| \\
\leq & C\left(\int_{G} w_{1}(y)^{t}|f(y)|^{t} w_{2}\left(y^{-1} x\right)^{t}\left|g\left(y^{-1} x\right)\right|^{t} \Delta^{\frac{t}{q}}\left(y^{-1} x\right) d_{L} \lambda(y)\right)^{1 / t}
\end{aligned}
$$

for $\lambda$-almost every $x \in G$. Under our assumptions on $p, q, r, t$, we have $\frac{1}{p / t}+$ $\frac{1}{q / t}=1$ and $\frac{1}{p / t}, \frac{1}{q / t}>1$. Thus, by a second application of Hölder's inequality we obtain

$$
\begin{aligned}
& w_{3}(x)\left|f * g \Delta^{1 / q}(x)\right| \\
\leq & C\left(\int_{G} w_{1}(y)^{p}|f(y)|^{p} d_{L} \lambda(y)\right)^{1 / p} \\
& \cdot\left(\int_{G} w_{2}\left(y^{-1} x\right)^{q}\left|g\left(y^{-1} x\right)\right|^{q} \Delta\left(y^{-1} x\right) d_{L} \lambda(y)\right)^{1 / q} \\
= & C\|f\|_{p, w_{1}}\|g\|_{q, w_{2}}
\end{aligned}
$$

for $\lambda$-almost every $x \in G$. Hence

$$
\|f * g\|_{\infty, w_{3}} \leq C\|f\|_{p, w_{1}}\|g\|_{q, w_{2}} .
$$

CASE V. Suppose that $p, q, r<\infty$, wherefrom $t<\infty$ as well. By Hölder's inequality and (4.4) we have

$$
\begin{aligned}
& \left|f * g \Delta^{\frac{1}{q}-\frac{1}{r}}(x)\right| \\
\leq & \int_{G} \frac{w_{1}(y)|f(y)| w_{2}\left(y^{-1} x\right)\left|g\left(y^{-1} x\right)\right| \Delta^{\frac{1}{q}-\frac{1}{r}}\left(y^{-1} x\right)}{w_{1}(y) w_{2}\left(y^{-1} x\right)} d_{L} \lambda(y) \\
\leq & \left(\int_{G} w_{1}(y)^{t}|f(y)|^{t} w_{2}\left(y^{-1} x\right)^{t}\left|g\left(y^{-1} x\right)\right|^{t} \Delta^{\frac{t}{q}-\frac{t}{r}}\left(y^{-1} x\right) d_{L} \lambda(y)\right)^{1 / t} \\
& \cdot\left(\int_{G} \frac{1}{w_{1}(y)^{t^{\prime}} w_{2}\left(y^{-1} x\right)^{t^{\prime}}} d_{L} \lambda(y)\right)^{1 / t^{\prime}} \\
= & \left(\int_{G} w_{1}(y)^{t}|f(y)|^{t} w_{2}\left(y^{-1} x\right)^{t}\left|g\left(y^{-1} x\right)\right|^{t} \Delta^{\frac{t}{q}-\frac{t}{r}}\left(y^{-1} x\right) d_{L} \lambda(y)\right)^{1 / t} \\
& \cdot\left(w_{1}^{-t^{\prime}} * w_{2}^{-t^{\prime}}(x)\right)^{1 / t^{\prime}} \\
\leq \quad & \left(\int_{G} w_{1}(y)^{t}|f(y)|^{t} w_{2}\left(y^{-1} x\right)^{t}\left|g\left(y^{-1} x\right)\right|^{t} \Delta^{\frac{t}{q}-\frac{t}{r}}\left(y^{-1} x\right) d_{L} \lambda(y)\right)^{1 / t} \\
& \cdot\left(C^{t^{\prime}} w_{3}^{-t^{\prime}}(x)\right)^{1 / t^{\prime}}
\end{aligned}
$$

Therefore

$$
\begin{aligned}
& w_{3}(x)\left|f * g \Delta^{\frac{1}{q}-\frac{1}{r}}(x)\right| \\
\leq & C\left(\int_{G} w_{1}(y)^{t}|f(y)|^{t} w_{2}\left(y^{-1} x\right)^{t}\left|g\left(y^{-1} x\right)\right|^{t} \Delta^{\frac{t}{q}-\frac{t}{r}}\left(y^{-1} x\right) d_{L} \lambda(y)\right)^{1 / t}
\end{aligned}
$$


Define

$$
F(x):=\left(w_{1}(x)|f(x)|\right)^{t} \quad \text { and } \quad G(x):=\left(w_{2}(x)|g(x)|\right)^{t} .
$$

Using this notation we can express 4.5 as follows :

$$
w_{3}(x)\left|f * g \Delta^{\frac{1}{q}-\frac{1}{r}}(x)\right| \leq C\left(F * G \Delta^{\frac{t}{q}-\frac{t}{r}}\right)(x)^{1 / t},
$$

SO

$$
\left\|f * g \Delta^{\frac{1}{q}-\frac{1}{r}}\right\|_{r, w_{3}}=\left\|w_{3}\left(f * g \Delta^{\frac{1}{q}-\frac{1}{r}}\right)\right\|_{r} \leq C\left\|F * G \Delta^{\frac{t}{q}-\frac{t}{r}}\right\|_{r / t}^{1 / t} .
$$

Note that we have $\frac{p}{t}, \frac{q}{t}, \frac{r}{t} \geq 1$ and

$$
\frac{1}{p / t}+\frac{1}{q / t}=\frac{1}{r / t}+1
$$

Therefore, Theorem 4.1 implies

$$
\left\|F * G \Delta^{\frac{1}{q / t}-\frac{1}{r / t}}\right\|_{r / t} \leq\|F\|_{p / t}\|G\|_{q / t}=\left\|w_{1} f\right\|_{p}^{t}\left\|w_{2} g\right\|_{q}^{t}=\|f\|_{p, w_{1}}^{t}\|g\|_{q, w_{2}}^{t} .
$$

Putting together 4.6 and 4.7) we obtain

$$
\left\|f * g \Delta^{\frac{1}{q}-\frac{1}{r}}\right\|_{r, w_{3}} \leq C\|f\|_{p, w_{1}}\|g\|_{q, w_{2}} .
$$

Note that under the stated assumptions on $p, q, r, t$, these five cases are exhaustive since (4.3) implies that we cannot have simultaneously $p=q=\infty$ and $r<\infty$; similarly when $p=\infty$ then $q$ and $r$ cannot be both finite; nor can $p$ and $r$ be both fini when $q=\infty$.

\section{Concluding remarks and questions}

Both Theorem 4.1 and Theorem 4.4 provide sufficient conditions for $\left(w_{1}, w_{2}, w_{3}\right)$ to belong to $\mathcal{Y}_{G}(p, q, r)$. A natural question to ask is whether these sufficient conditions are necessary.

One can easily show (using the Dirac measure at $x$ ) that if $G$ is discrete, then $\left(w_{1}, w_{2}, w_{3}\right) \in \mathcal{Y}_{G}(p, q, r)$ only if condition 4.2 holds true. This means, in the case where $G$ is discrete and $p, q, r \in[1, \infty]$ satisfy 4.1), that condition 4.2 completely characterizes the class $\mathcal{Y}_{G}(p, q, r)$. If, instead, $p, q, r$ satisfy 4.3 , then condition 4.2 is not sufficient; for an easy explicit counterexample, consider $G:=\mathbb{Z}, w_{1}=w_{2}=w_{3} \equiv 1, p=q=r=t=2, \alpha \in\left(\frac{1}{2}, \frac{3}{4}\right)$, and the function $f: \mathbb{Z} \rightarrow \mathbb{C}$ defined by

$$
f(k):= \begin{cases}k^{\alpha}, & k>0 \\ 0, & k \leq 0\end{cases}
$$


Clearly $f \in \ell_{2}(\mathbb{Z}) \cong \ell_{2}\left(\mathbb{Z}, w_{1}\right) \cong \ell_{2}\left(\mathbb{Z}, w_{2}\right)$. On the other hand

$$
(f * f)(n)=\sum_{k=1}^{n} k^{-\alpha}(n-k)^{-\alpha} \geq \sum_{k=1}^{n} k^{-\alpha} n^{-\alpha} \geq C_{\alpha} n^{1-\alpha} n-\alpha
$$

for all $n \geq 1$, where $C_{\alpha}>0$ is a constant independent of $n$. As $\alpha<\frac{3}{4}$, it follows that $f * f \notin \ell_{2}(\mathbb{Z}) \cong \ell_{2}\left(\mathbb{Z}, w_{3}\right)$.

As for condition (4.4), one can readily verify that it completely characterizes the class $\mathcal{Y}_{G}(p, q, r)$ if $p=q=r=t=\infty$. Under the additional assumption that $G$ be compact, we can even relax the assumptions on $p, q, r, t$ and simply require that $r=\infty$ and 4.3 holds true.

Remark that the case where $p, q, r, t<\infty$ verify 4.3 stands out. Indeed, in [23, 26], Kuznetsova provided various counterexamples showing that the condition 4.2 is not necessary for the the conclusion of Theorem 4.4 to hold true. Nevertheless, the following question remains open.

Question 5.1. If $1<p, q, r, t<\infty$ verify the hypothesis of Theorem 4.4 , does there exist a countable abelian group $G$ and weight functions $w_{1}, w_{2}, w_{3}$ on $G$ such that $\left(w_{1}, w_{2}, w_{3}\right) \in \mathcal{Y}_{G}(p, q, r)$ but 4.4 fails ?

\section{Acknowledgement}

The author is grateful to Professors Javad Mashreghi and Thomas J. Ransford for their helpful comments and suggestions. The author would like to thank the referee for his/her valuable and insightful comments. The paper has benefited substantially from them.

\section{References}

[1] Abtahi, F., Nasr-Isfahani, R., And Rejali, A. Weighted $L^{p}$-conjecture for locally compact groups. Period. Math. Hungar. 60, 1 (2010), 1-11.

[2] Beckner, W. Inequalities in Fourier analysis on $R^{n}$. Proc. Nat. Acad. Sci. U.S.A. 72 (1975), 638-641.

[3] Bergh, J., And Löfström, J. Interpolation spaces. An introduction. SpringerVerlag, Berlin-New York, 1976. Grundlehren der Mathematischen Wissenschaften, No. 223.

[4] Biswas, A., And Swanson, D. Navier-Stokes equations and weighted convolution inequalities in groups. Comm. Partial Differential Equations 35, 4 (2010), $559-589$.

[5] Blanco, A., Kaijser, S., And Ransford, T. J. Real interpolation of Banach algebras and factorization of weakly compact homomorphisms. J. Funct. Anal. 217, 1 (2004), 126-141.

[6] Brascamp, H. J., And Lieb, E. H. Best constants in Young's inequality, its converse, and its generalization to more than three functions. Advances in Math. 20, 2 (1976), 151-173. 
[7] Edwards, R. E. The stability of weighted Lebesgue spaces. Trans. Amer. Math. Soc. 93 (1959), 369-394.

[8] El-Fallah, O., And Ransford, T. Peripheral point spectrum and growth of powers of operators. J. Operator Theory 52, 1 (2004), 89-101.

[9] Feichtinger, H. G. Gewichtsfunktionen auf lokalkompakten Gruppen. Österreich. Akad. Wiss. Math.-Natur. Kl. Sitzungsber. II 188, 8-10 (1979), 451471.

[10] Fendler, G., Gröchenig, K., And Leinert, M. Symmetry of weighted $L^{1}$ algebras and the GRS-condition. Bull. London Math. Soc. 38, 4 (2006), 625-635.

[11] Fournier, J. J. F. Sharpness in Young's inequality for convolution. Pacific J. Math. 72, 2 (1977), 383-397.

[12] Gabardo, J.-P. Sampling and interpolation in weighted $L^{2}$-spaces of bandlimited functions. Sampl. Theory Signal Image Process. 17, 2 (2018), 197-224.

[13] Grabiner, S. Weighted convolution algebras as analogues of Banach algebras of power series. In Radical Banach algebras and automatic continuity (Long Beach, Calif., 1981), vol. 975 of Lecture Notes in Math. Springer, Berlin-New York, 1983, pp. 282-289.

[14] Gröchenig, K. Weight functions in time-frequency analysis. In Pseudodifferential operators: partial differential equations and time-frequency analysis, vol. 52 of Fields Inst. Commun. Amer. Math. Soc., Providence, RI, 2007, pp. 343-366.

[15] Gröchenig, K., And Leinert, M. Wiener's lemma for twisted convolution and Gabor frames. J. Amer. Math. Soc. 17, 1 (2004), 1-18.

[16] Gröchenig, K., And Leinert, M. Symmetry and inverse-closedness of matrix algebras and functional calculus for infinite matrices. Trans. Amer. Math. Soc. 358, 6 (2006), 2695-2711.

[17] Guo, W., Chen, J., Fan, D., and Zhao, G. Characterizations of some properties on weighted modulation and Wiener amalgam spaces. Michigan Math. J. 68,3 (2019), 451-482.

[18] Hewitt, E., And Ross, K. A. Abstract harmonic analysis. Vol. I, second ed., vol. 115 of Grundlehren der Mathematischen Wissenschaften [Fundamental Principles of Mathematical Sciences]. Springer-Verlag, Berlin-New York, 1979. Structure of topological groups, integration theory, group representations.

[19] Kerlin, E., And Lambert, A. Strictly cyclic shifts on $l_{p}$. Acta Sci. Math. (Szeged) 35 (1973), 87-94.

[20] Kerman, R., And SAWyer, E. Convolution algebras with weighted rearrangement-invariant norm. Studia Math. 108, 2 (1994), 103-126.

[21] Klein, A., And Russo, B. Sharp inequalities for Weyl operators and Heisenberg groups. Math. Ann. 235, 2 (1978), 175-194.

[22] Kuznetsova, Y., And Molitor-Braun, C. Harmonic analysis of weighted $L^{p}$-algebras. Expo. Math. 30, 2 (2012), 124-153.

[23] Kuznetsova, Y. N. Weighted $L_{p}$-algebras on groups. Funktsional. Anal. $i$ Prilozhen. 40, 3 (2006), 82-85.

[24] Kuznetsova, Y. N. The invariant weighted algebras $l_{p}^{w}(g)$. Mat. Zametki 84, 4 (2008), 567-576. 
[25] Kuznetsova, Y. N. Constructions of the regular algebras $L_{p}^{w}(G)$. Mat. Sb. 200, 2 (2009), 75-88.

[26] Kuznetsova, Y. N. Example of a weighted algebra $L_{p}^{w}(G)$ on an uncountable discrete group. J. Math. Anal. Appl. 353, 2 (2009), 660-665.

[27] Nikol'skiı̆, N. K. Selected problems of weighted approximation and spectral analysis. American Mathematical Society, Providence, R.I., 1976. Translated from the Russian by F. A. Cezus.

[28] Toft, J., Johansson, K., Pilipović, S., And Teofanov, N. Sharp convolution and multiplication estimates in weighted spaces. Anal. Appl. (Singap.) 13, 5 (2015), 457-480.

[29] Wermer, J. On a class of normed rings. Ark. Mat. 2 (1954), 537-551.

Received by the editors September 24, 2019

First published online February 10, 2020 